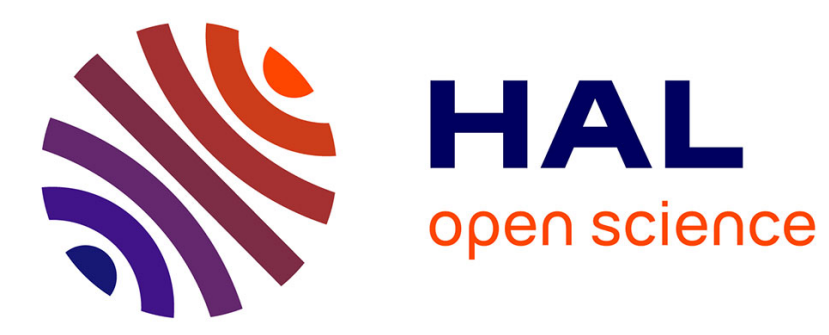

\title{
Charges électriques convectées par des écoulements hautement turbulents
}

\author{
L. Marcano, G. Touchard, M. F. Morin
}

\section{To cite this version:}

L. Marcano, G. Touchard, M. F. Morin. Charges électriques convectées par des écoulements hautement turbulents. Revue de Physique Appliquée, 1987, 22 (9), pp.1081-1085. 10.1051/rphysap:019870022090108100 . jpa-00245647

\section{HAL Id: jpa-00245647 https://hal.science/jpa-00245647}

Submitted on 1 Jan 1987

HAL is a multi-disciplinary open access archive for the deposit and dissemination of scientific research documents, whether they are published or not. The documents may come from teaching and research institutions in France or abroad, or from public or private research centers.
L'archive ouverte pluridisciplinaire HAL, est destinée au dépôt et à la diffusion de documents scientifiques de niveau recherche, publiés ou non, émanant des établissements d'enseignement et de recherche français ou étrangers, des laboratoires publics ou privés. 


\title{
Charges électriques convectées par des écoulements hautement turbulents
}

\author{
L. Marcano, G. Touchard et M.F. Morin \\ L.E.A., U.A. 191 du C.N.R.S., Laboratoire de Physique et Mécanique des Fluides, 40 avenue du Recteur \\ Pineau, 86022 Poitiers, France
}

(Reçu le 24 novembre 1986, révisé le 5 mars 1987, accepté le 11 juin 1987)

\begin{abstract}
Résumé.- La première partie de cette étude présente deux manières différentes d'aborder théoriquement le phénomène de transports de charges dans des écoulements turbulents : une basée sur le rapport entre la vitesse fluctuante radiale de l'écoulement et la vitesse de relaxation de la couche diffuse ; l'autre, basée sur la diffusité turbulente dont les modèles théoriques ont été jusqu'alors très grossiers. C'est pourquoi, nous nous sommes attachés à redéfinir correctement une telle modélisation. Pour celà, le profil de diffusivité est ensuite déterminé et les équations à résoudre dans le cas où théoriquement le phénomène d'électrisation est supposé contrôlé par la diffusivité turbulente sont explicitées. La deuxième partie décrit une étude expérimentale sur les courants d'écoulements dans le cas du G.N.L. (Gaz Naturel Liquéfié), qui nous permettra de vérifier la validité des prévisions théoriques.
\end{abstract}

\begin{abstract}
In the first part of this study we present two different models of the phenomenon of streaming current in turbulent flows. One is based on the ratio between the fluctuante radial velocity of the flow with the relaxation velocity of the diffuse layer; the other one takes into account the eddy diffusity, but the theoretical studies already published with this model being always very rough, we tried in this paper to elaborate another theory from this modelisation. Thus the eddy diffusivity profile is settled and the equations of the charge transport established. The experimental work is related in the second part of the study for flows of L.N.G. (Liquefied Natural Gas). At least the streaming currents measured are compared with the theoretical predictions.
\end{abstract}

\section{Introduction}

Lors du transfert de grandes quantités de gaz liquéfiés, les potentiels électriques générés, dûs à l'électrisation, peuvent être très importants (plusieurs centaines de kilovolts à en juger par les étincelles observées). L'importance de ces potentiels généralement supérieurs à ceux observés pour les hydrocarbures est principalement dûe à un temps de relaxation plus grand pour ces liquides (leur résistivité électrique étant très élevée) et à leur viscosité très faible donnant lieu à des écoulements fortement turbulents. Du point de vue appliqué, l'intérêt de l'étude de ces phénomènes est évident sur le plan de la sécurité. Du point de vue fondamental, il est aussi extrêmement intéressant car il est possible d'atteindre les écoulements avec des nombres de Reynolds très élevés, même à l'intérieur de conduites de diamètre relativement petit. C'est la possibilité de tester, dans un domaine jusqu'alors pratiquement totalement vierge, les différentes théories élaborées pour le transport de charges par des écoulements turbulents.

\section{Etude théorique}

Lors d'un écoulement turbulent, il y a perturbation de la couche diffuse, donc, de la densité volumique de charges. Ces perturbations ơnt été interprétées théoriquement par deux théories différentes.

Une première étude, réalisée par $\mathrm{G}$. Touchard [1], considère que dans un écoulement turbulent, la couche diffuse présente deux régions :

- l'une près de la paroi, non perturbée, où la densité volumique de charges reste celle existante au repos ;

- l'autre, située dans la partie centrale de la conduite, complètement perturbée; dans cette région la densité volumique de charges est uniformisée. 
Le point de raccordement est lié au rapport entre la vitesse fluctuante radiale et la vitesse de relaxation (Fig.1). La somme globale de charges dans la couche diffuse est par ailleurs supposée constante.

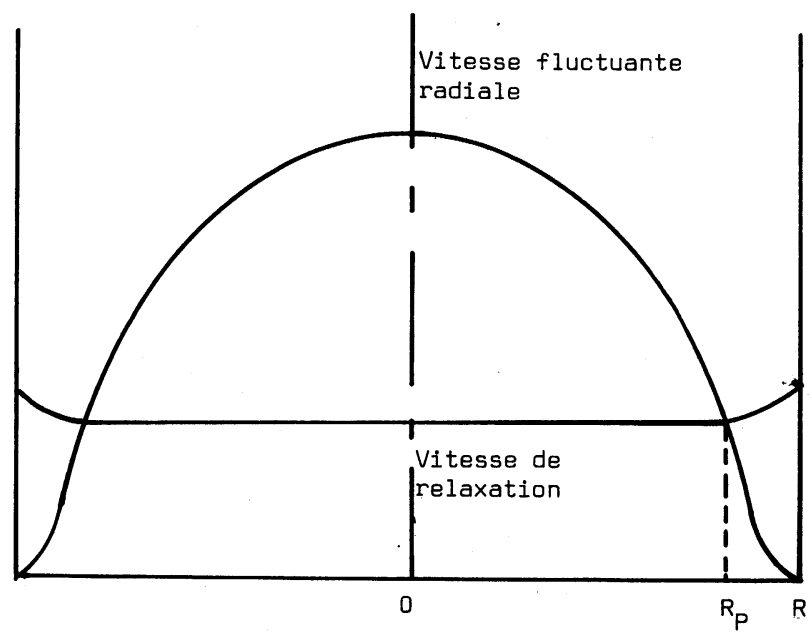

Fig.1.- Distribution de la vitesse fluctuante radiale et de la vitesse de relaxation.

[Distribution of the radial velocity fluctuation and of the relaxation velocity.]

La confrontation faite par G. Touchard [2] entre cette modélisation et les résultats expérimentaux obtenus pour l'hexane et l'heptane montre une assez bonne concordance théorie-expériences. Cependant, d'un point de vue fondamental il est intéressant de bien analyser la différence entre les prévisions théoriques données par cette modélisation, et celle tenant compte de la diffusivité turbulente. Des expériences réalisées pour des grands nombres de Reynolds devraient alors permettre de déterminer le domaine de validité des différentes hypothèses faites pour ces deux modélisations.

Dans une étude ultérieure B. Abedian [3], suppose que les grandeurs qui interviennent dans le phénomène de transport de charges, ont une composante fluctuante et il fait apparaître le coefficient de diffusivité turbulente.

Malheureusement B. Abedian ne fait qu'une étude très grossière du phénomène, en se limitant à des cas asymptotiques (faibles densités) et en utilisant des représentations des variables hydrodynamiques très approchées.

Par exemple, l'expression utilisée pour le profil de diffusivité turbulente est très loin des résultats expérimentaux trouvés dans la littérature [4-7] comme nous pouvons le constater figure 2 , courbe 1 (DT est le coefficient de diffusivité turbulente, $u_{*}$ la vitesse de frottement, $R$ le rayon du tube et $Y$ la distance à la paroi).

C'est pourquoi, si nous voulons faire une comparaison sur des bases correctes des deux modélisations précédentes, nous devons chercher tout d'abord une

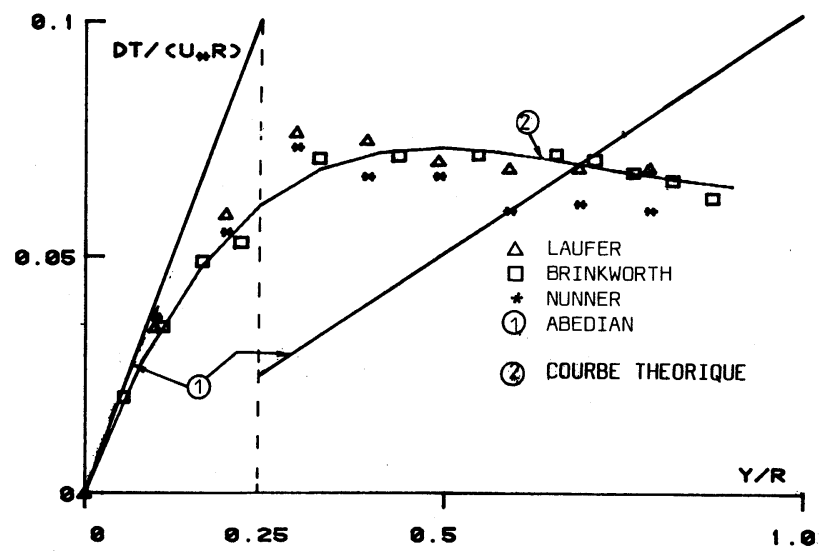

Fig.2.- Evolution de la diffusivité turbulente avec la distance à la paroi.

[Evolution of the eddy diffusivity in terms of the distance to the pipe wall.]

meilleure représentation du profil de diffusivité turbulente, car de lui dépend le calcul de la charge convectée.

2.1 PROFIL DE DIFFUSIVITE TURBULENTE.-La détermination du profil de diffusivité turbulente nécessite une analyse relativement complexe que nous expliciterons dans une prochaine publication. Cependant nous pouvons d'ores et déjà montrer les résultats obtenus pour la variation du coefficient de diffusivité turbulente en fonction de la distance à la paroi (Fig.2, courbe 2). Nous voyons que l'accord avec les résultats expérimentaux est correcte. Cette expression du coefficient de diffusivité turbulente nous permet alors de calculer la charge transportée.

2.2 CourANTS D'ECOULEMENTS.- L'intensité de courant adimensionnel est donné par l'intégrale :

$$
I_{+}=\int_{0}^{R_{+}} 2 \pi \rho_{+} u_{+} r_{+} \mathrm{d} r_{+}
$$

Où $R_{+}$est le rayon adimensionnel $\left(R_{+}=R / \delta, \delta\right.$ étant l'épaisseur de la couche diffuse), $\rho_{+}$est la densité volumique de charges adimensionnelle.

$\rho_{+}$vérifiant par ailleurs le système d'équations suivant :

$$
\begin{aligned}
\left(\left(1+D T_{+}\right)^{2}-\right. & \left.\beta^{2} D T_{+}^{2}\right) \frac{\mathrm{d} \rho_{+}}{\mathrm{d} r_{+}}+ \\
& +\left(D T_{+}\left(\sqrt{\rho_{+}^{2}+\alpha^{4}}+\beta \rho_{+}\right)+\right. \\
& \left.+\sqrt{\rho_{+}^{2}+\alpha^{4}}\right) \frac{\mathrm{d} \psi_{+}}{\mathrm{d} r_{+}}=0 \\
& \frac{\mathrm{d}^{2} \psi_{+}}{\mathrm{d} r_{+}^{2}}+\frac{1 \mathrm{~d} \psi_{+}}{r_{+} \mathrm{d} r_{+}}=-\rho_{+}
\end{aligned}
$$

Où $D T_{+}$est la diffusivité turbulente adimensionnelle $\left(D T_{+}=D T / D_{0}, D_{0}\right.$ représente le coefficient 
de diffusion moyen), $\psi_{+}$est le potentiel électrique adimensionnel, $\alpha$ et $\beta$ sont des coefficients adimensionnels :

$$
\alpha^{2}=1-\beta^{2} \text { et } \beta=\left(D_{\mathrm{P}}-D_{\mathrm{N}}\right) /\left(D_{\mathrm{P}}+D_{\mathrm{N}}\right)
$$

$D_{\mathrm{P}}$ et $D_{\mathrm{N}}$ étant les coefficients de diffusion des cations et des anions.

Des résultats complets de l'évolution des courants d'écoulement calculés à partir de cette modélisation feront l'objet de la publication prochaine déjà citée. Nous pouvons cependant dire, à partir d'une analyse sommaire des résultats obtenus, que dans bien des cas le courant calculé est proportionnel aux nombres de Reynolds. Ce résultat est semblable à celui prévu par la modélisation faite par G. Touchard tenant compte de la vitesse fluctuante radiale, mais semble en désaccord avec les prévisions faites par B. Abedian.

\section{Etude expérimentale}

3.1 MONTAGE EXPERIMENTAL.- Un schéma général du montage est donné figure 3 . Ce montage est comparable à celui décrit précédemment [8], mais avec un certain nombre de modification nous permettant de travailler en circuit fermé et de mesurer le débit grâce à une sonde de niveau.

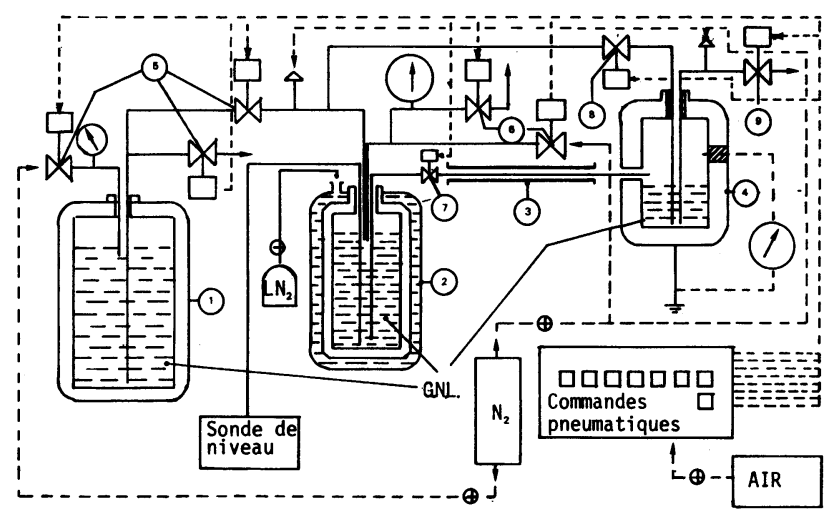

Fig.3.- Montage expérimental. 1) Récipient de stockage. 2) Récipient d'alimentation. 3) Tube capillaire. 4) Récipient collecteur. 5,6,7,8) Vannes pneumatiques.

[The experimental equipment. 1) Storage vessel, 2) Pressurisation vessel, 3) Capillary tube, 4) Collecting vessel, $5,6,7,8)$ Pneumatic gates.]

3.2 EXPERIENCES.- Nous avons testé plusieurs sortes de G.N.L. de compositions chimiques différentes et nous avons fait des expériences pour des pressions variant entre 0 et 2 bars. Trois conduites de un mètre de long et $1,16 \mathrm{~mm} ; 2 \mathrm{~mm}$ et $2,5 \mathrm{~mm}$ de diamètre ont été utilisées.

Nous avons fait deux séries d'expériences avec du G.N.L. ayant un taux de méthane de $74 \%$ et de REVUE DE PHYSIQUE APPLIQUÉE. - T. 22, N`9, SEPTEMBRE 1987
$88,4 \%$. Cependant, avant de présenter les résultats obtenus pour les courants d'écoulement, nous devons signaler l'extrême difficulté à effectuer de telles expériences, liées à la fois aux très faibles points d'ébullition de tels liquides, aux risques que présentent les manipulations dans le cas du G.N.L. et bien sûr, au fait que toute expérience réalisée sur des courants d'écoulement conduit à mesurer des courants très faibles et souvent liés aux conditions hygrométriques de l'environnement.

Un phénomène supplémentaire est venu perturber nos expériences. En effet, nous avons remarqué que malgré l'excellent isolement thermique des différents cryostats, le fluide s'écoulait à l'état diphasique à la sortie du capillaire. Cet état diphasique venant modifier nos valeurs expérimentales, nous avons dû tenir compte de ce nouveau paramètre et notamment estimer la fraction de liquide dans l'écoulement.

3.2.1. Calcul de la fraction massique de liquide convecté.- Pour un fluide diphasique, nous pouvons poser en première approximation :

$\rho_{\mathrm{m}}=X \rho_{1}+(1-X) \rho_{\mathrm{g}} ; \mu_{\mathrm{m}}=X \mu_{1}+(1-X) \mu_{\mathrm{g}}$

Où $X$ représente le volume de liquide dans un volume unitaire de fluide, $\rho_{\mathrm{m}}, \rho_{\mathrm{l}}, \rho_{\mathrm{g}}$ sont les densités volumiques du fluide, du liquide et du gaz, $\mu_{\mathrm{m}}$, $\mu_{1}, \mu_{\mathrm{g}}$ sont les viscosités dynamiques du fluide, du liquide et du gaz. Lorsqu'on est en présence d'un écoulement turbulent, nous avons :

$\Delta P=\frac{0,3164 \mu_{\mathrm{m}}^{2} L}{16 \rho_{\mathrm{m}} R^{3}} \operatorname{Re}^{7 / 4}$ et $Q=\frac{\pi}{2} \operatorname{Re}\left(\mu_{\mathrm{m}} / \rho_{\mathrm{m}}\right) R$

$\Delta P$ est la différence de pression entre les extrémités de la conduite, $R$ et $L$ le rayon et la longueur de la conduite.

$Q$ est le débit volumique du fluide $\left(Q_{1}=X Q\right.$, $Q_{1}$ étant le débit de liquide).

A partir des équations ci-dessous, nous pouvons écrire :

$$
Q_{1}=K \Delta P^{4 \Pi}
$$

Le coefficient $K$ peut être déterminé à partir d'une représentation $\log$-Log des valeurs expérimentales obtenues pour $Q_{1}$, et $X$ est alors obtenu par résolution numérique de l'équation :

$$
\begin{aligned}
& \left(X \rho_{1}+(1-X) \rho_{\mathrm{g}}\right)^{3 / 7} \times \\
& \times\left(X \mu_{1}+(1-X) \mu_{\mathrm{g}}\right)^{1 / 7} \frac{2 K}{\pi X}=\left(\frac{16}{0,3164}\right)^{4 / 7} \frac{R^{19 / 7}}{L^{4 / 7}}
\end{aligned}
$$

$X$ connu, nous pouvons estimer la fraction massique de liquide $y_{\mathrm{m}}$, sachant que :

$$
y_{\mathrm{m}}=X \rho_{1} /\left(X \rho_{1}+(1-X) \rho_{\mathrm{g}}\right)
$$

Nous obtenons finalement pour le G.N.L. à $74 \%$ et 
à $88,4 \%$ en méthane, les valeurs consignées dans le tableau I.

Tableau I.

\begin{tabular}{c|c|c|c}
$R(\mathrm{~mm})$ & 0,58 & 1 & 1,24 \\
$y_{\mathrm{m}}$ & & & \\
\hline G.N.L. à $74 \% \mathrm{CH}_{4}$ & 0,594 & 0,853 & 0,819 \\
G.N.L. à $88,4 \% \mathrm{CH}_{4}$ & 0,998 & 0,999 & 0,999
\end{tabular}

Nous voyons que les écoulements de G.N.L. à 74 $\%$ étaient fortement diphasiques, c'est pourquoi nous ne présentons ici que les résultats obtenus pour les courants d'écoulements dans le cas du G.N.L. à $88,4 \%$.

3.2.2. Courants d'écoulements.- Les figures 4 à 6 rassemblent les résultats obtenus pour les courants d'écoulements dans le G.N.L. à 88,4\% en méthane.

Malgré la dispersion des résultats dûe notamment aux très grandes difficultés expérimentales, nous constatons que, pour les trois conduites étudiées, le courant d'écoulement est grossièrement proportionnel aux nombres de Reynolds comme prévu théoriquement, et que par conséquent la densité volumique de charges convectées semble constante pour un écoulement turbulent quelle que soit la vitesse d'écoulement.

Des renseignements plus précis sur ce phénomène dans le cas du G.N.L. sont à l'heure actuelle guère possible à obtenir vues les difficultés expérimentales à surmonter. Une analyse plus complète pourra être faite sur d'autre liquides cryogéniques dont les conditions expérimentales sont plus facilement contrôlables. Toutefois, du point de vue de la sécurité, il est important de signaler que pratique-

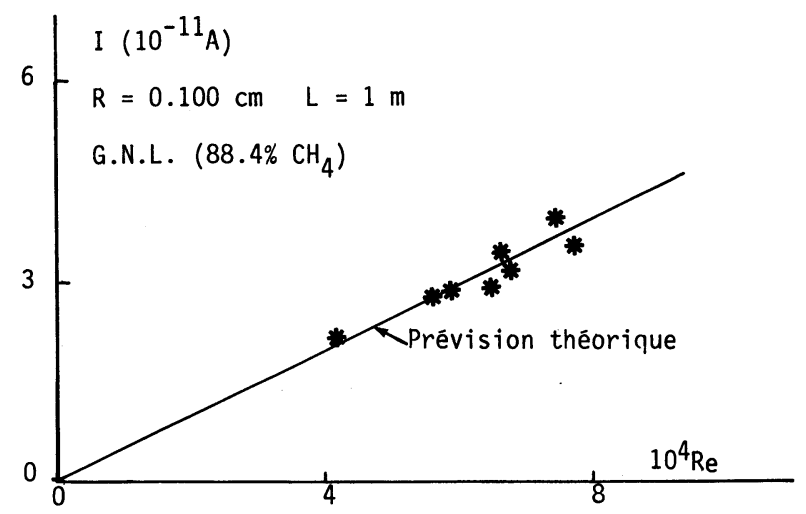

Fig.4.- Evolution du courant d'écoulement en fonction du nombre de Reynolds.

[Streaming current variation in terms of Reynolds number.]

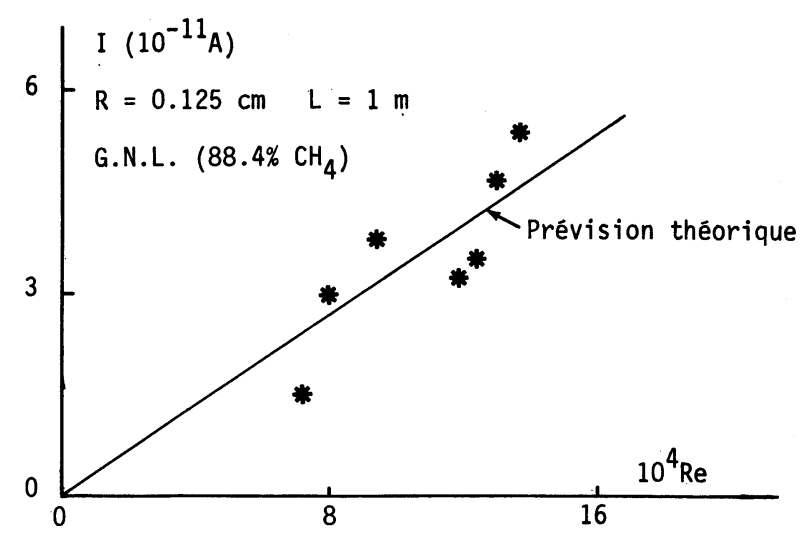

Fig.5.- Evolution du courant d'écoulement en fonction du nombre de Reynolds.

[Streaming current variation in terms of Reynolds number.]

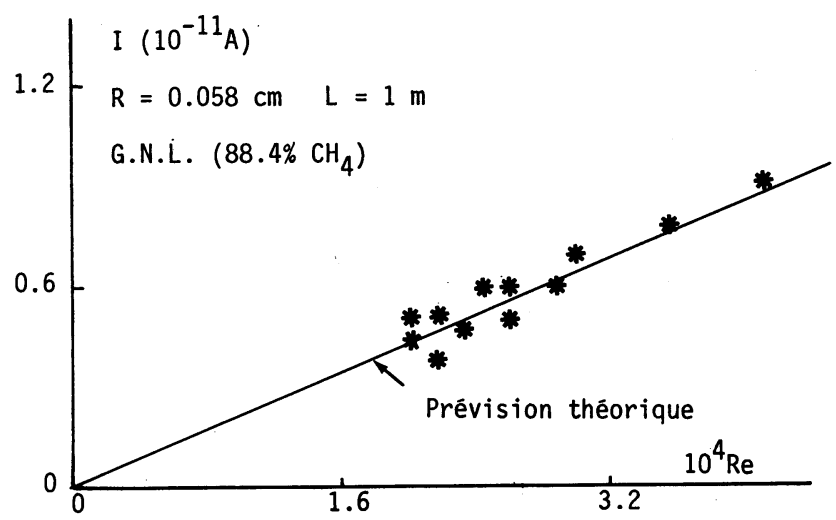

Fig.6.- Evolution du courant d'écoulement en fonction du nombre de Reynolds.

[Streaming current variation in terms of Reynolds number.]

ment, dans toutes les expériences faites avec le G.N.L., bien que les valeurs obtenues pour les courants d'écoulement aient été généralement inférieures à celles que l'on observe pour d'autres hydrocarbures, les densités volumiques de charges générées semblent suffisantes pour produire des potentiels importants lors du remplissage des réservoirs de grandes dimensions, étant donné le temps de relaxation de ces liquides. Ceci explique vraisemblablement les étincelles parfois observées.

\section{Conclusion}

Dans cette étude, nous avons, d'une part présenté des résultats théoriques concernant la modélisation du phénomène de transport de charges dans des écoulements hautement turbulents, notamment l'effet et l'allure de la diffusivité turbulente au sein de tels écoulements.

D'autre part, des résultats expérimentaux obtenus 
avec du G.N.L. montrent que l'influence de l'aspect diphasique du fluide est très importante. Cependant la comparaison entre les valeurs expérimentales des courants d'écoulements obtenus pour trois conduites différentes avec les prévisions données par une approche trés simplifiée de la théorie basée sur le phénomène de diffusivité turbulente, est globalement satisfaisante. Il serait maintenant intéressant de comparer une étude plus complète s'appuyant sur cette théorie avec celle élaborée par G. Touchard.

\section{Bibliographie}

[1] Touchard, G. et Dumargue, P., J. Electroanal. Chem. 88 (1978) 387-405.

[2] Toúchard, G. et Dumargue, P., J. Electrostatics 14 (1983) 209-223.

[3] Abedian, B. et Sonin, A., J. Fluid Mech. 120 (1982) 199-217.

[4] LAufer, J., NACA Rep. (1954) 1174.
[5] Hinze, J.O., Turbulence (Mc Graw-Hill, New-York) (1959) 503-527.

[6] Brinkworth, B.J. et SMITH, P.C., Chem. Eng. Sci. 28 (1973) 1847-1852.

[7] Quarmby, A. et ANAND, R.K., J. Fluid Mech. 38 (1969) 433-455.

[8] Marcano, L. et Touchard, G., J. Electrostatics 15 (1984) 321-328. 